\title{
Schistosoma Mansoni Proctocolitis with Polyposis
}

\author{
Fridah Bosire, Ian Orwa, Michael Mwachiro, Robert Parker, Russell White
}

Tenwek Hospital

Correspondence to: Dr. Fridah Bosire, P.O. Box 39 Bomet, Kenya. Email: fridahbosire@gmail.com

\begin{abstract}
Summary
Schistosoma proctocolitis is a rare disease entity, with limited literature on its occurrence. We report the case of a 17 year old male from an endemic area around Lake Victoria, Western Kenya, where detailed disease mapping of Schistosomiasis has been carried out. The patient presented with a seven year history of a protruding rectal mass and occasional hematochezia. Colonoscopy revealed multiple polyps involving the entire colon to the anus, and pathology showed inflammatory polyps secondary to Schistosoma
\end{abstract}

mansoni. He was successfully managed with medical therapy.

Key words: Schistosomiasis, Helminthiasis, Neglected Diseases, Colonoscopy, Colonic Polyps

Ann Afr Surg. 2018; 15(2):81-85

DOI: http://dx.doi.org/10.4314/aas.v15i2.11

(C) 2018 Author. This work is licensed under the

Creative Commons Attribution 4.0 International

License.

Conflicts of Interest: None

Funding: None

\section{Introduction}

Schistosomiasis or Bilharziasis is a chronic infection caused by blood flukes. This parasitic disease constitutes significant public health challenges, and is associated with great morbidity and mortality with millions of people being affected worldwide. Globally, at least 230 million people are infected $(1,2,14)$. According to a World Health Organization (WHO) report in 2014, the disease is prevalent in 74 countries, with at least 52 endemic countries including Kenya where preventative strategies have been set in place. Over 258 million people required preventive treatment and 62 million were treated for schistosomiasis (14). Ninety percent of these cases were in Africa $(2,5)$.

In Kenya, the annual mortality rate from schistosomiasis has decreased by $61.3 \%$ since 1990 $(10,11)$, majorly due to public health strategies put in place that target chemotherapy given to school going children. The disease is endemic throughout the entire region. Estimates for the proportion of the population infected in Kenya have remained at $23 \%$ since the first estimation in1986 [11]. According to WHO, 12 million people in Kenya require treatment annually for schistosomiasis, but only 1 million people were treated in 2013. This makes up only $15 \%$ of the population in need. School-age children make up nearly half of the infected population in Kenya.

Schistosomiasis frequently impacts impoverished rural communities near fresh water bodies, with poor hygiene standards $(2,12)$. Agrarian and fishing regions are thus more affected (10, 12). Particularly vulnerable populations include women who do domestic chores in infested water bodies and children who bathe/swim in them. Infection occurs after direct contact with fresh water that contains the larval form of the parasite, also called cercaria (1, 2, and 13). Humans are the definitive hosts where the parasite undergoes sexual reproduction, and snails are the intermediate hosts $(12,13)$.There are different species identified, with the intestinal form (Schistosoma mansoni) being the most prevalent in East Africa along the shores of Lake Victoria $(10,11)$, as well as adjacent islands, and on the Kano Plain (Bondo, Kisumu West, Kisumu East, Nyando, Suba, Rachuonyo and Homa Bay in Nyanza Province). The disease is also endemic in rest of Africa, South America, Caribbean and Middle East. We report a case of Schistosoma 
mansoni proctocolitis in a 17 year old from Homa Bay, Kenya.

\section{Case Presentation}

A 17-year-old HIV- negative male from Homa Bay, Western Kenya, presented with a seven-year history of protruding rectal mass necessitating manual reduction, with associated occasional fresh per rectal bleeding that he quantified to about four times a week. He experienced intermittent episodes of diarrhoea and denied a history of weight loss, abdominal pain, jaundice or hematuria. He reported a history of blood transfusion for attendant anaemia four years after his symptoms started. He had no family history of colorectal malignancy, with no smoking or alcohol intake history. He had not used any type of medication, had no chronic illnesses, and had no prior history of surgery or colonoscopy. He came from a rural fishermen family along the shores of Lake Victoria, and the family relied on water from the shores of the lake for most domestic chores. Other than mild pallor, the patient had a normal physical exam, with no signs of liver disease. Digital rectal exam revealed normal tone with soft palpable rectal protrusions, with no blood on examining finger. Blood tests done, including liver function tests, were normal, except for mild microcytic hypochromic anaemia with Hemoglobin of $11.8 \mathrm{~g} / \mathrm{dL}$. Due to the diarrhoea, stool tests were ordered. Stool microscopy with saline wet mount preparations revealed Entamoeba histolytica trophozoites, and he was treated for this with oral metronidazole, with subsequent resolution of the symptom. Repeat stool tests on follow up demonstrated no Entamoeba histolytica cysts or trophozoites. Colonoscopy demonstrated multiple polyps from the ileo-cecal valve to the ano-cutaneous line: some flat, sessile, pedunculated; the largest one at $3 \mathrm{~cm}$ in size. Biopsies were taken from the colon and rectum. Pathological examination demonstrated both inflammatory hyperplastic changes with marked eosinophilia and eosinophilic abscesses and the rectal polyp having viable schistostosoma eggs with identified lateral spines consistent with S. Mansoni (Figure 2). Medical management was initiated. The patient was treated with Praziquantel 2.4gm in two divided doses for 1 day $(40 \mathrm{mg} / \mathrm{kg} /$ day). Using his weight of 60kilograms, Praziquantel dosage was calculated as $2400 \mathrm{mg} /$ day, given in two divided dosages (1200mg BID). Retreatment was done after four weeks using the same dosage. Scheduled clinic visits at eight weeks showed improvement of symptoms, with resolution of per rectal bleeding.

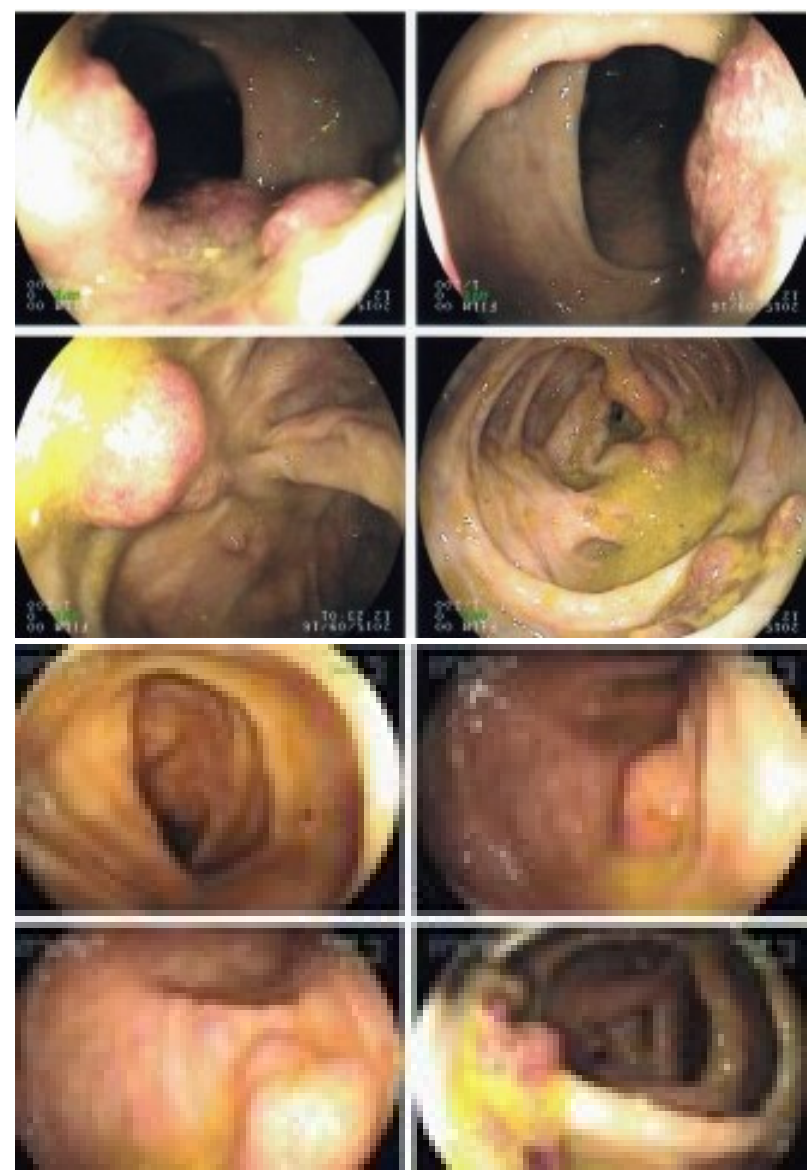

Figure 1: Colonoscopy images showing multiple polyps

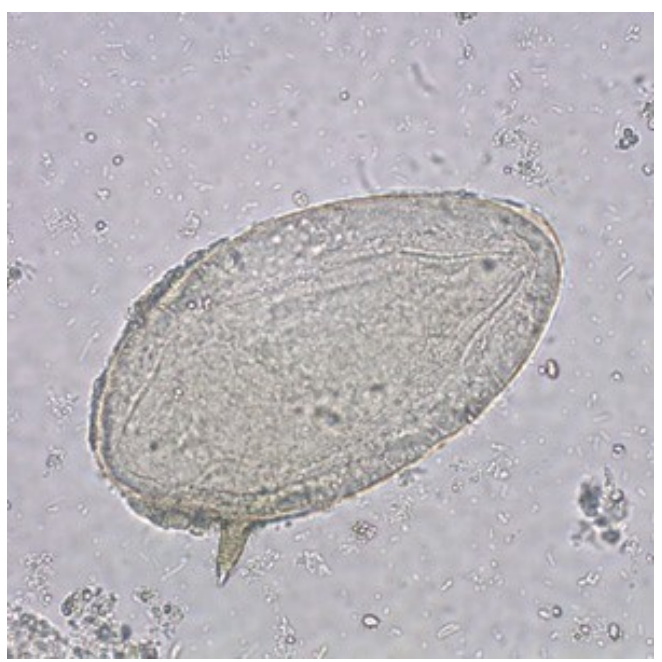

Figure 2: Egg of Schistosoma mansoni with characteristic lateral spine 


\section{Discussion}

Schistosoma proctocolitis is a rare disease entity, with limited literature on its occurrence. This was a case report study on Schistosomal proctocolitis and polyposis, which must be considered within the differential diagnosis of gastrointestinal disease when treating patients from endemic areas $(8,15)$. We presented a 17-year-old male from an endemic area in Nyanza, Kenya, who presented with protruding rectal mass with per rectal bleeding. Colonoscopy revealed multiple polyps from the ano cutaneous line to the ileo-cecal valve, and biopsies taken came back as inflammatory hyperplastic changes with viable Schistosoma mansoni eggs. This case highlights the need for surgeons and endoscopists to be aware of the potential sequelae of neglected tropical diseases in patients from endemic areas.

S. mansoni prevalence in Kenya is stated as $13 \%$. This is due to Biomphalaria species of snails found around Lake Victoria $(11,12)$. Our patient came from Homa Bay, along the shores of the lake. As a referral center for endoscopy and surgery and with increasing travel among Kenyan patients, it is important to consider diseases endemic to other regions within Kenya.

Pathogenesis of the disease entails understanding the life cycle of the micro-organism, and how it ends up in tissues. Schistosoma mansoni is most commonly implicated in intestinal disease, especially in the colon $(4,8,9)$. Patients may present with non-specific symptoms, such as intermittent diarrhoea, abdominal pain, and per rectal bleeding. Thus differential diagnosis may entail inflammatory bowel disease and/or colorectal malignancy. Symptom frequency is often affected by intensity of the infection, and the spectrum of intestinal schistosomiasis may vary from granulomatous disease to diffuse proctocolitis with polyposis $(8,15)$, as was in the case of our patient. The extended duration of symptoms such as found in this patient who is from a resource-limited setting could have contributed to the development and severity of polyposis. From the life cycle above, viable eggs containing miracidia can implant onto the bowel wall, causing inflammation with activation of various immune cells. A giant cell reaction occurs, and the ensuing inflammation causes edema and congestion,

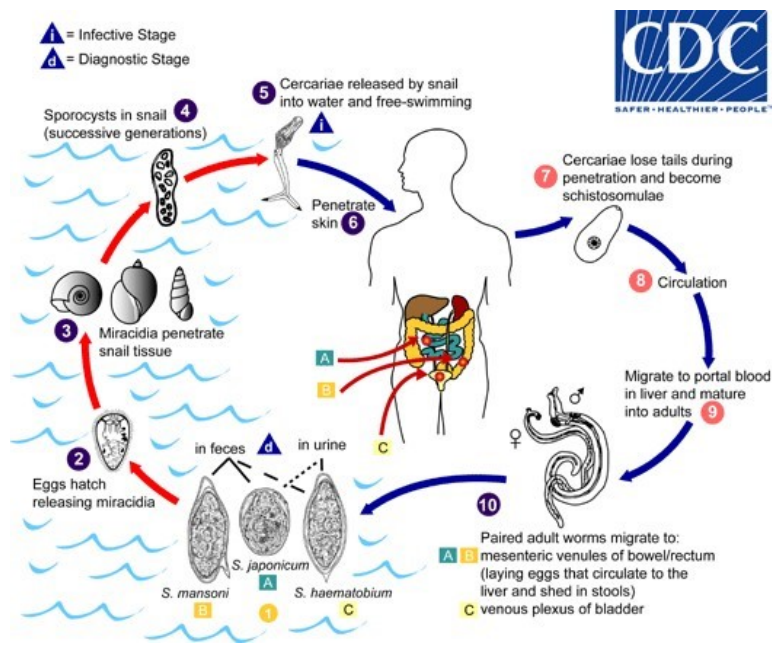

Figure 3: Life Cycle

with ultimate formation of granulomas on the bowel wall. This can result in polyp formation, or even ulceration.

Procto-colonoscopic examination is key in establishing the diagnosis of Schistosomal proctocolitis (8), alongside stool tests and tissue diagnosis with biopsy. Colonoscopy findings include hyperaemic mucosa with thickening, edema, granulomas, ulceration, and rarely inflammatory polyps which may be sessile or pedunculated $(4,9)$, and are seen in chronic infections as was the case in our patient. All sections of the colon may be affected, but over $90 \%$ of cases involve the inferior mesenteric vein territory, with the descending colon most frequently involved $(4,6)$.

Limited studies exist on pre-malignant potential of these infections. Schistosoma japonica has been linked to colorectal malignancy in populations in China and Japan $(16,17)$. The studies have however been inconclusive, with limited direct causal inference. Although evidence for the relationship between Schistosoma mansoni and colorectal cancer is limited (18), the potential for malignancy was discussed with the patient presented and recommendations made to undergo surveillance until complete resolution of polyposis. Conclusions about malignant potential are limited due to the lack of reports in the literature (18). Clinical suspicion for schistosomiasis should be high in areas that are endemic. Elimination of the flukes forms the major objective of preventative strategies set in place by $\mathrm{WHO}$, that majorly entail mass drug administration to schools and communities $(5,12)$. 
Management can either be pharmacologic through medical therapy, or surgical. Medical therapy is considered first line, and literature supports that abnormal colonoscopy findings may resolve with medical treatment $(2,5)$. The drug of choice is Praziquantel, an antihelminthic, usually given as a single dose with cure rates of $60-95 \%(10,11,13)$. Its mechanism of action is not clearly understood, but is thought to work by increasing the permeability of schistosome cell membranes to calcium ions (11). This induces contraction of the parasite and leaves it paralyzed. The parasites are then cleared by phagocytosis. It is usually given as an oral single dose of $40-60 \mathrm{mg} / \mathrm{kg} /$ day $(1,9)$. Retreatment at two to six weeks has been shown to increase cure rates. Surgical therapy has a role in severe or chronic cases refractory to medical treatment. It may include resection of the colonic polyps, or partial colectomy for gastrointestinal polyposis $(9,15)$.

Medical therapy is thus crucial and recommended in the prevention and management of intestinal schistosomiasis. Public health initiatives can target this, and provide preventative chemoprophylaxis to people living in endemic areas, as well as create awareness on the disease. For surgeons and endoscopists, this is an important differential diagnosis for patients presenting with abdominal pains, per rectal mass, bleeding and polyposis on colonoscopy.

\section{Conclusion}

Schistosoma mansoni proctocolitis is a rare entity, but of clinical significance not only to public health initiative programs but to the surgeon as well. High index of clinical suspicion is important in clinical set ups that serve endemic areas. As demonstrated in this case report, the patient, once diagnosed, can be successfully managed with medical treatment.

\section{Acknowledgements}

We thank the parents of the patient described for allowing us to work on this and share his details, as well as giving informed written consent for the research work and subsequent follow up.

\section{References}

1. Ross A, Bartley P, Sleigh AC, et al. Schistosomiasis. New Engl J Med. 2002, 346(16):1212-20.

2. Barsoum R, Esmat G, El Baz T. Human Schistosomiasis: Clinical Perspective: Review. J Adv Res. 2013.

3. Centre of Disease Control. Schistosomiasis. CDC DPDx 2013 Jun [cited 2017Jul 20]. Available from:

https://www.cdc.gov/dpdx/schistosomiasis/index.h tml

4. Lamyman MJ, Noble DJ, Narang S, et al. Small Bowel Obstruction Secondary to Intestinal Schistosomiasis. Trans R Soc Trop Med Hyg. 2006, 100 (9): 885-7.

5. Kabatereine NB, Fleming FM, Nyandindi U, et al. The Control of Schistosomiasis and Soil Transmitted Helminths in East Africa. Trends Parasitol 2006, 22 (7):332-9.

6. Mazigo H, Chandika A, Zinga M., et al. Intestinal Schistosomiasis Associated with Intususception: A Case Report. Tanzan J Health Res. 2011.

7. Mazigo HD, Giiti GC, Zinga M, et al. Schistosomal Peritonitis Secondary to Perforated Appendicitis. Braz J Infect Dis2010, 14 (6):62830.

8. Cao J, Liu WJ, Xu XY, et al. Endoscopic Findings and Clinicopathologic Characteristics of Colonic Schistosomiasis: A Report of 46 Cases. World J Gastroenterol. 2010, 16 (6): 723-7.

9. Al Moferreh M, Ahmed I, Tandon R, et al. Endoscopic Polypectomy in Colorectal Schistosomiasis at Riyadh Central Hospital. Ann Saudi Med 1988; 8: 131-4

10. Odiere M, Rawago F, Ombok M, et al. High Prevalence of Schistosomiasis in Mbita and its Adjacent Islands of Lake Victoria, Western Kenya. Parasit Vectors. 2012, 5: 278.

11. Malenganisho WL, Magnussen P, Friis H, et al. Schistosoma Mansoni Morbidity Among Adults in Two Villages Along Lake Victoria Shores in Mwanza District, Tanzania. Trans R Soc Trop Med Hyg 2008, 102 (6):532-41 
12. Ofulla AV, Adoka SO, Anyona DN, et al. Spatial Distribution and Habitat Characterization of Schistosomiasis Host Snails in Lake and Land Habitats of Western Kenya. Lakes Res Res Manage. 2013, 18: 197-215.

13. Opisa S, Odiere M, Jura W, et al. Malacological Survey and Geographical Distribution of Vector Snails for Schistosomiasis Within Informal Settlements of Kisumu City, Western Kenya. Parasit Vectors. 2011, 4: 226-10.

14. "Schistosomiasis Fact Sheet". World Health Organization. 2016.

15. Meybehm M, Dworák O, Gugler R:

Granulomatous Colitis with Pseudopolyp in Schistosomiasis. Dtsch Med Wochenschr. 1989, 114 (1): 19-22

16. Hashimoto Y, Muratani A, Nishiyama H, et al. A Case of Colon Cancer Associated with Schistosomiasis Japonica. Gan No Rinsho. 1986, 32 (7): 815-8.

17. Li WC, Pan ZG, Sun YH. Sigmoid Colonic Carcinoma Associated with Deposited Ova of Schistosoma Japonicum: A Case Report. World J Gastroenterol. 2006, 12 (37): 6077-9.

18. Salim OE, Hamid HK, Mekki SO, et al. Colorectal Carcinoma Associated with Schistosomiasis: A Possible Causal Relationship. World J Surg Oncol. 2010, 8:68. 